\title{
The interaction of dendritic cells and $\gamma \delta$ T cells promotes the activation of $\gamma \delta$ T cells in experimental autoimmune uveitis
}

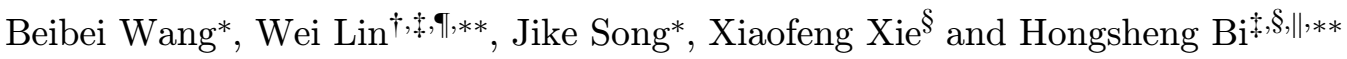 \\ *Shandong University of Traditional Chinese Medicine \\ 4655\#, Daxue Road, Jinan City 250355, P. R. China \\ ${ }^{\dagger}$ Department of Microbiology \\ Shandong Academy of Medical Sciences \\ Jinan City 250030, P. R. China \\ Eye Institute of Shandong University of Traditional Chinese Medicine \\ 48\#, Yingxiongshan Road, Jinan City 250002, P. R. China \\ $\S_{\text {Affiliated Eye Hospital }}$ \\ Shandong University of Traditional Chinese Medicine \\ 48\#, Yingxiongshan Road, Jinan City 250002, P. R. China \\ 9linw1978@163.com \\ "hongshengbi@126.com
}

Received 30 December 2015

Accepted 15 May 2016

Published 13 July 2016

\begin{abstract}
Uveitis is a severe inflammatory disease that can cause visual impairment. Recently, activated $\gamma \delta$ $\mathrm{T}$ cells were proved to play a central role in the development of experimental autoimmune uveitis (EAU). However, the mechanism underlying $\gamma \delta$ T-cell activation in EAU is incompletely known. In this study, we determined the percentage changes in and the phenotypes of $\gamma \delta \mathrm{T}$ cells and dendritic cells (DCs) obtained from the spleens of immunized C57BL/6 (B6) mice, an animal model of EAU. We found that the number of $\gamma \delta \mathrm{T}$ cells and DCs obviously increased during the inflammation phase of EAU (days 16-20 of our experiment), and that during this time, $\gamma \delta \mathrm{T}$ cells expressed high levels of CD69 and the integrin lymphocyte function-associated antigen-1 (LFA1) and secreted high levels of interleukin (IL)-17A. Moreover, DCs obtained during this phase expressed high levels of CD80, CD83, CD86, and intracellular cell adhesion molecule-1 (ICAM-1). Furthermore, we studied the interaction between DCs and $\gamma \delta$ T cells by using flow cytometry and confocal microscopy in order to determine whether DCs affected $\gamma \delta$ T-cell activation in vitro. Co-cultures of the two types of cells showed that DCs induced high levels of CD69, LFA-1, and
\end{abstract}

\footnotetext{
${ }^{* *}$ Corresponding authors.
}

This is an Open Access article published by World Scientific Publishing Company. It is distributed under the terms of the Creative Commons Attribution 4.0 (CC-BY) License. Further distribution of this work is permitted, provided the original work is properly cited. 
IL-17A in $\gamma \delta \mathrm{T}$ cells. Imaging studies revealed contact between the DCs and $\gamma \delta \mathrm{T}$ cells. This interaction was mediated by the accumulation of ICAM- 1 and LFA- 1 at the interface of DCs- $\gamma \delta$ $\mathrm{T}$ cells. Thus, the activation of $\gamma \delta \mathrm{T}$ cells in EAU was promoted by DCs interacting with $\gamma \delta$ $\mathrm{T}$ cells.

Keywords: $\gamma \delta \mathrm{T}$ cells; dendritic cells; experimental autoimmune uveitis; integrin lymphocyte function-associated antigen-1 (LFA-1).

\section{Introduction}

Uveitis is a serious inflammatory disease that can cause visual impairment. This condition usually occurs in young adults and children. ${ }^{1} \mathrm{~A}$ disorder of the immune system is considered to be the key factor in the occurrence of uveitis. However, the exact mechanism underlying the development of uveitis remains unclear. Recently, many reports have shown that $\gamma \delta \mathrm{T}$ cells play an important role in the regulation and resolution of inflammatory processes associated with infections, tumors, and autoimmunity diseases. ${ }^{2-4}$ Moreover, $\gamma \delta \mathrm{T}$ cells can have either an up-regulation or a down-regulation effect on adaptive immune responses. ${ }^{5-9}$ Studies have shown that $\gamma \delta \mathrm{T}$ cells play a central role in the development of experimental autoimmune uveitis (EAU), and the effect of $\gamma \delta$ T cells critically depends on their state of activation. ${ }^{10,11}$ Activated $\gamma \delta$ T cells promote the activation of $\mathrm{IL}-17^{+}$autoreactive $\mathrm{T}$ cells to enhance EAU development, whereas nonactivated $\gamma \delta \mathrm{T}$ cells have little effect on EAU. ${ }^{10,11}$ However, the mechanism of $\gamma \delta \mathrm{T}$ cells activation in EAU is still incompletely understood. Although antigen and cytokines might promote the activation of $\gamma \delta \mathrm{T}$ cells, the function of interactions between $\gamma \delta$ T cells and other immune cells on activation of $\gamma \delta \mathrm{T}$ cells has not been well defined. Herein, we studied whether $\gamma \delta \mathrm{T}$ cells were activated by interacting with dendritic cells (DCs), which were an initiator of adaptive immune responses.

DCs are the most powerful antigen-presenting cells (APCs) and they initiate and regulate a broad repertoire of immune responses. ${ }^{12,13}$ DCs are the link between innate and adaptive immunity. They have an immense capacity to regulate T-cell activation. ${ }^{14,15}$ They also interact with innate lymphocytes such as natural killer (NK) cells, NK T cells, and $\gamma \delta \mathrm{T}$ cells to regulate innate immunity. DCs promote the secretion of cytokines and the activation of innate lymphocytes, which in turn regulate the maturation of DCs. ${ }^{16,17}$ Mature DCs highly express major histocompatibility complex (MHC) and co-stimulatory molecules such as intracellular cell adhesion molecule-1 (ICAM-1), CD40, CD80, and CD86, and secrete interleukin (IL)-12. ${ }^{18,19} \mathrm{DCs}$ are believed to be an initiator of adaptive immune responses in some diseases, such as EAU. Some reports have shown that the number, phenotype, and function of DCs are altered in EAU, and thereby participate in the development of EAU. ${ }^{20,21}$ The aim of the present study was to determine whether DCs participate in the development of EAU by regulating the activation of $\gamma \delta \mathrm{T}$ cells.

In this study, we constructed an EAU model to detect the number and phenotypes of DCs and $\gamma \delta \mathrm{T}$ cells during the "inflammatory phase" of EAU (days 16-20 of our experiment), and determined the effects of DCs on $\gamma \delta$ T cell activation. Furthermore, by using flow cytometry and confocal microscopy, we analyzed the mechanism of DC promoted $\gamma \delta$ T-cell activation. Our results suggested that the interactions between DCs and $\gamma \delta \mathrm{T}$ cells play a major role in the regulation of $\gamma \delta$ T-cell activation.

\section{Materials and Methods}

\subsection{Animals and reagents}

Pathogen-free female C57BL/6 Mice (B6) (6 to 8-weeks-old) were purchased from Peking Vital River Laboratory Animal Ltd., Beijing, China and were fed and maintained in specific pathogen-free conditions according to the guidelines of Care and Use of Laboratory Animals published by the China National Institute of Health. $\gamma \delta$ TCR- $\delta^{-/-}$mice $(8$ weeks old) were purchased from Jackson laboratory (Bar harbor, ME, USA). All experimental procedures adhered to the Association for Research in Vision and Ophthalmology Statement for the use of animals in ophthalmic and vision research. The human interphotoreceptor retinoid-binding protein peptide (IRBP $)_{1-20}$ was synthesized by ChinaPeptides Co., Ltd., Shanghai, China. Complete Freund's adjuvant (CFA) was obtained from Sigma 
(St. Louis, MO, USA). Pertussis toxin (PTX) was purchased from Enzo Life Sciences (Farmingdale, YN, USA). Fluorescein antibodies of CD3e, MHCII, CD11c, IL-17A, CD69 and LFA-1 were purchased from eBioscience (San Diego, CA, USA). PE-anti-mouse TCR $\gamma \delta$ was obtained from BD Biosciences (San Jose, CA, USA). Anti-TCR gamma and TCR delta antibody [UC7-13D5] (Phycoerythrin), anti-CD11c antibody [N418] and goat anti-armenian hamster IgG H\&L (Alexa Fluor 647) were purchased from Abcam (Cambridge, MA, USA). Recombinant murine IL-2 and IL-23 were purchased from R\&D Systems (Minneapolis, MN, USA). CD11c + isolated kit, $\gamma \delta \mathrm{T}$ cells isolated kit were purchased from Miltenyi Biotec (Miltenyi Biotec, Bergisch Gladbach, Germany).

\subsection{Induction and evaluation of $E A U$}

B6 mice were immunized subcutaneously at six spots (on the footpads, tail base, and flank) with $200 \mu \mathrm{l}$ of an emulsion containing $300 \mu \mathrm{g} \mathrm{IRBP}_{1-20}$ emulsified in CFA. Concurrently, a single dose of 500 ng PTX was injected intraperitoneally.

After immunization, the mice were examined for clinical signs of EAU on days 0, 4, 8, 12, 16, 20, 24, 28,32 , and 36 by using a Genesis-D camera (Kowa Company Ltd., Hamamatsu City, Japan). The disease was graded using scoring systems based on acknowledged and modified criteria, ${ }^{22}$ as displayed in Supplementary Table 1.

In addition, inflammation in the eye was confirmed by histopathologic examination. Eyes were obtained from the immunized mice on days $0,4,8$, $12,16,20,24,28,32$, and 36 , and were fixed for $48 \mathrm{~h}$ in $4 \%$ buffered glutaraldehyde. The fixed tissues were embedded in paraffin, sectioned $(4-6 \mu \mathrm{m})$ through the papillary-optic nerve plane, and stained with hematoxylin and eosin $(\mathrm{H} \& \mathrm{E})$. They were observed under a microscope ( $\mathrm{Ti}$, Nikon, Japan), and the disease was graded on the basis of cellular infiltration and structural changes, which have been described in Supplementary Table 2.

\subsection{Purification of DCs and $\gamma \delta T$ cells}

DCs were purified from the spleens of normal or $\mathrm{IRBP}_{1-20}$-immunized $\mathrm{B} 6$ mice, by using $\mathrm{CD} 11 \mathrm{c}+$ Microbeads and an autoMACS separator (both from Miltenyi Biotec, Bergisch Gladbach, Germany), according to the manufacturer's instructions.
Similarly, $\gamma \delta$ T cells were purified from the spleens of $\mathrm{IRBP}_{1-20}-$-immunized or normal $\mathrm{B} 6$ mice by using a $\gamma \delta$ T-cell isolation kit (Miltenyi Biotec, Bergisch Gladbach, Germany). In brief, for $\gamma \delta$ T-cell isolation, $1 \times 10^{8}$ lymphocytes were incubated with $50 \mu \mathrm{l}$ Non-T-Cell Depletion Cocktail in $450 \mu$ l phosphatebuffered saline (PBS) at $4^{\circ} \mathrm{C}$ for $15 \mathrm{~min}$. The cells were then washed once with PBS. The cells were resuspended in PBS and loaded on an autoMACS separation column, and $50 \mu \mathrm{l}$ Anti-Biotin MicroBeads in $450 \mu \mathrm{l}$ PBS were added. The resuspended cells were separated at $4{ }^{\circ} \mathrm{C}$ for $15 \mathrm{~min}$. The purity of the isolated cells was determined using flow cytometry (Supplementary Fig. 1).

\subsection{Analysis of $\gamma \delta T$ cell and $D C$ phenotypes}

Splenic cells were obtained from normal mice and EAU mice on days $4,8,12,16,20,24,28,32$, and 36 after immunization. $\mathrm{T}$ cells were isolated from the spleen by passage through a nylon wool column. The cells were then collected by Ficoll-Hypaque density gradient centrifugation, and cultured at $37^{\circ} \mathrm{C}$ in a carbon dioxide incubator for another $12 \mathrm{~h}$. The obtained cells were stained by direct immunofluorescence and analyzed using flow cytometry (FACSVerse; BD Biosciences, USA). To determine the number of $\gamma \delta \mathrm{T}$ cells and their activation status, we stained aliquots of $1 \times 10^{6} \mathrm{~T}$ cells with phycoerythrin (PE)-conjugated $\gamma \delta$ T-cell receptor (TCR) and APC-conjugated CD3e antibodies, or with PEconjugated lymphocyte function-associated antigen-1 (LFA-1) and fluorescein isothiocyanate (FITC)-conjugated CD69. To determine the number of DCs, we stained splenic cells with PEconjugated MHC-II and FITC-conjugated CD11c antibodies. Next, to analyze the phenotype of DCs, we stained the DCs with APC-conjugated ICAM-1, CD80, CD83, or CD86. Data collection and analysis were performed using a FACSVerse flow cytometer and CellQuest software.

\subsection{Assessment of the interaction between DCs and $\gamma \delta$ T cells in vitro by flow cytometry}

We purified $\gamma \delta \mathrm{T}$ cells and DCs from the spleens of immunized mice by using autoMACS. The $\gamma \delta$ $\mathrm{T}$ cells were incubated at $37^{\circ} \mathrm{C}$ in RPMI-1640 
B. Wang et al.

(a)

(b)

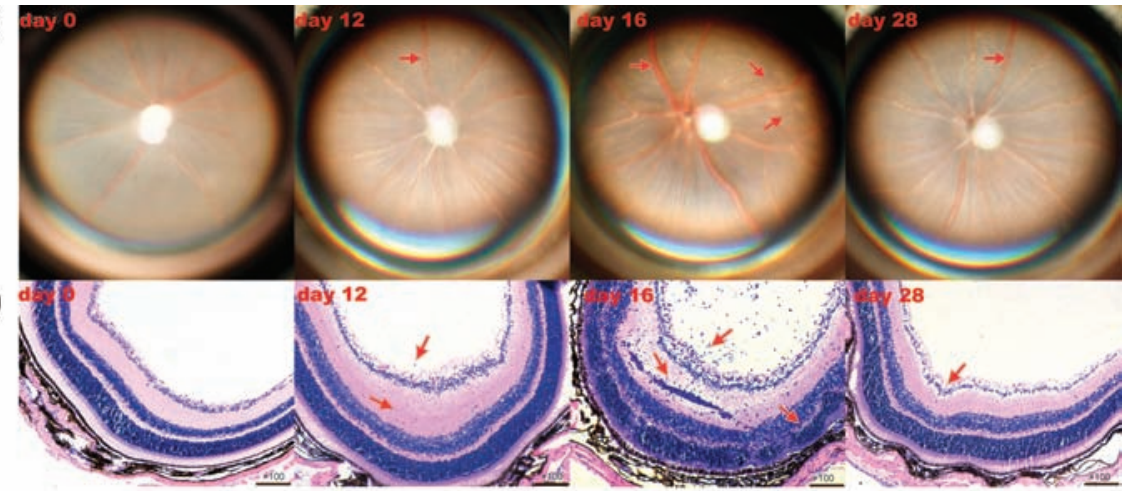

(c)

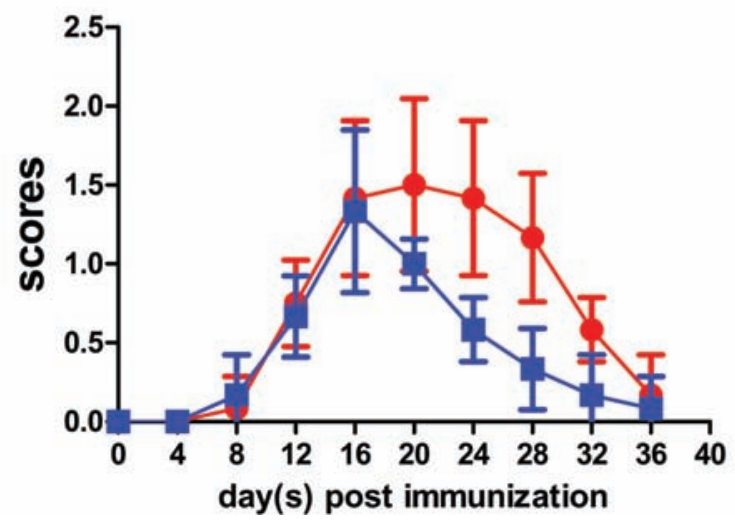

Fig. 1. EAU model creation. (a) B6 mice were immunized subcutaneously with $\mathrm{IRBP}_{1-20}$, and disease symptoms were evaluated by fundoscopy. Minimal vasculitis was observed on day 12. Multifocal chorioretinal lesions, severe vasculitis, and linear lesions were observed on days 16-20. Ocular inflammation was gradually resolved on day 28. (b) Histopathology of a representative eye section from control (day 0) and $\mathrm{IRBP}_{1-20}$-immunized mice on days 12,16 , and 28 (hematoxylin and eosin; original magnification, $\times 100$ ). Image of a representative eye on day 16 after immunization (in the severe inflammation phase) shows heavy cell infiltration and retinal folds (arrows). (c) EAU scores after immunization. The data presented are the average scores of three separate experiments. The results are expressed as mean $\pm \mathrm{SD}(n=6)$.

medium in 24-well plates coated with IL-2 (10 ng/ $\mathrm{ml})$ and IL-23 $(10 \mathrm{ng} / \mathrm{ml})$. DCs were stimulated with IL-4 $(20 \mathrm{ng} / \mathrm{ml})$ and granulocyte macrophage colony-stimulating factor $(100 \mathrm{ng} / \mathrm{ml})$. Lipopolysaccharide $(200 \mathrm{ng} / \mathrm{ml})$ to promote maturation. Mature and immature DCs were washed and incubated for $2 \mathrm{~h}$ in the above medium supplemented with $\operatorname{IRBP}_{1-20}(20 \mathrm{ng} / \mathrm{ml})$. Then, mature and immature DCs were separately co-cultured with $\gamma \delta \mathrm{T}$ cells at a $1: 1$ ratio in 24 -well plates, at $37^{\circ} \mathrm{C}$. After $24 \mathrm{~h}$, the cells were harvested and stained with PEconjugated LFA-1, FITC-conjugated CD69, and APC-conjugated IL-17A. Activated $\gamma \delta \mathrm{T}$ cells were prepared by incubating the isolated $\gamma \delta \mathrm{T}$ cells with $\operatorname{IRBP}_{1-20}(20 \mathrm{ng} / \mathrm{ml})$, IL-2 $(10 \mathrm{ng} / \mathrm{ml})$ and IL-23 $(10 \mathrm{ng} / \mathrm{ml})$ for $24 \mathrm{~h}$. With anti-ICAM-1 antibody treatment, anti-ICAM-1 antibody $(10 \mu \mathrm{g} / \mathrm{ml})$ pretreated the DCs for $2 \mathrm{~h}$ and then pulsed IRPB $_{1-20}$ to DCs for $2 \mathrm{~h}$ and $\gamma \delta \mathrm{T}$ cells were added to co-culture with DCs.

\subsection{Flow cytometric analysis}

Aliquots of $1 \times 10^{6}$ cells were stained with different monoclonal antibodies. After being incubated for $30 \mathrm{~min}$ and washed twice, cells from each sample were analyzed using FACSVerse and the CellQuest data acquisition and analysis software (BD Biosciences, USA). To assess intracellular cytokine expression, we stimulated the prepared cells for $5 \mathrm{~h}$ with leukocyte activation cocktail (BD Biosciences, USA) at $37^{\circ} \mathrm{C}$ in a $5 \% \mathrm{CO}_{2}$ environment. The cells were then harvested and transferred to tubes, washed once with PBS, and incubated with APC-conjugated IL-17A antibody 
after fixation and permeabilization, according to the manufacturer's instructions.

\subsection{Confocal microscopy assessment of $\gamma \delta$ T-cell-DC interaction}

DCs and $\gamma \delta \mathrm{T}$ cells were purified from the spleens of the IRBP ${ }_{1-20}$-immunized $\mathrm{B} 6$ mice. The $\gamma \delta \mathrm{T}$ cells were incubated with $\mathrm{IRBP}_{1-20}$-pulsed mature DCs for $30 \mathrm{~min}$, until $\gamma \delta$ T-cell-DC contact had been established. Then, all the cells were fixed in PBS/ $4 \%$ paraformaldehyde for $10 \mathrm{~min}$, followed by incubation with $\mathrm{PBS} / 0.1 \mathrm{M}$ glycine for $3 \mathrm{~min}$ and blocking with PBS $/ 2 \%$ bovine serum albumin buffer for $20 \mathrm{~min}$. Next, the cells were stained with a 1:10 dilution of PE-conjugated anti- $\gamma \delta$ TCR antibody and APC-conjugated ICAM-1 antibody for $60 \mathrm{~min}$. After being washed three times, the cells were observed using confocal microscopy.

The spleen from the immunized mice was frozen in O.C.T. Compound (VWR scientific Products, So. Plainfield, NJ). Seven-micrometer- thick spleen sections were cut, fixed and then were permeabilized with $0.1 \%$ Triton-X-100 for $10 \mathrm{~min}$, and blocked with $5 \%$ serum for $1 \mathrm{~h}$ at $25^{\circ} \mathrm{C}$, Anti-mouse CD11c antibody at a dilution of 1:20 was incubated overnight at $4^{\circ} \mathrm{C}$ in a humidified chamber, cells were washed with PBS and incubated with secondary antibody (1:200) at room temperature in the dark. Then anti-TCR $\gamma / \delta$ antibody (phycoerythrin) (1:50) was incubated overnight at $4^{\circ} \mathrm{C}$. Niclei were counterstained with Hoechst (1:10000).

Images of the cells were taken with a confocal microscope (LMS 780, Zeiss, Germany) equipped with an APO oil immersion objective lens $(63 \times$, $\mathrm{NA}=1.40$ ). To quantify the redistribution of molecules at the contact site, $\gamma \delta$ T-cell-DC doublets were chosen from bright-field images, and only $\gamma \delta$ T-cell-DC pairs whose contact orientation was proper for the $x-y$ plane projection were taken into consideration for further analysis. The images were analyzed with the Imaris software (Bitplane AG, Zurich, Switzerland).

\subsection{Statistical analysis}

The data analysis was performed by SPSS 17.0 software (SPSS, Chicago, IL, USA). Each experiment was carried out in duplicate and repeated three times. Data were represented as the mean \pm standard deviation.

\section{Results}

\subsection{Increase in the number of $\gamma \delta$ T cells and DCs and their activation in the spleen in the inflammatory phase of $\boldsymbol{E A} \boldsymbol{U}$}

We prepared the EAU model in C57BL/6 mice to analyze the station of $\gamma \delta \mathrm{T}$ cells in EAU development. EAU was successfully induced by $\operatorname{IRBP}_{1-20}$ in C57BL/6 mice (Fig. 1). Compared to the normal eye, the diseased eye showed increased blood vessel growth and inflammatory cell infiltration in the vitreous and retinal disorganization. The inflammatory symptoms in the eyes appeared on day 12 of the experiment. The initial changes were fundal blood vessel thickening and minimal inflammatory cell infiltration. Then, multifocal chorioretinal lesions, severe vasculitis, and linear lesions were observed on days 16-20, along with abundant lymphocyte infiltration in the vitreous and retinal disorganization. The inflammatory symptoms in the eyes began to abate from day 28 onwards. Representative images of the changes are shown in Figs. 1(a) and 1(b). Clinical scores were recorded using a Genesis-D camera, and histological scores were obtained from $\mathrm{H} \& \mathrm{E}$ analysis (Fig. 1(c)). The clinical and histological scores peaked on days 1620 , indicating that the severity of the inflammation was greatest during this period, which we have termed as the "inflammatory phase" in this study. We studied the changes in $\gamma \delta$ T cells and DCs in the inflammatory phase in the immunized mice, and compared these findings with those observed in the normal mice during the same period.

Using flow cytometry, we determined the number of $\gamma \delta$ T cells and DCs obtained from the spleens of the normal and immunized mice. In the normal mice, only $1.58 \% \pm 0.2 \%$ of the splenic $\mathrm{T}$ cells were $\gamma \delta \mathrm{T}$ cells, but in the immunized mice in the inflammatory phase, $5.62 \% \pm 1.09 \%$ of the splenic $\mathrm{T}$ cells were $\gamma \delta \mathrm{T}$ cells (Fig. 2(a), left column, each figure shows a representative result of three experiments). In the normal mice, $2.19 \% \pm 0.13 \%$ of the splenic cells were DCs, whereas in the immunized mice in the inflammatory phase, $9.77 \% \pm$ $1.91 \%$ of splenic cells were DCs (Fig. 2(a), right column, each figure shows a representative result of three experiments). Furthermore, the $\gamma \delta \mathrm{T}$ cells harvested from the immunized mice in the inflammatory phase expressed higher levels of the surface 


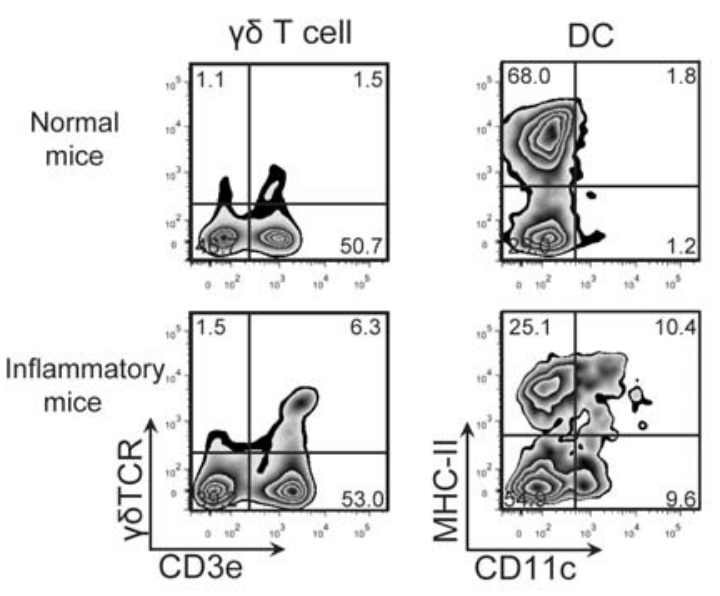

(a)
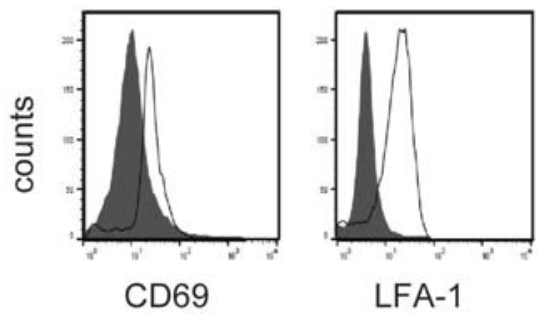

(b)
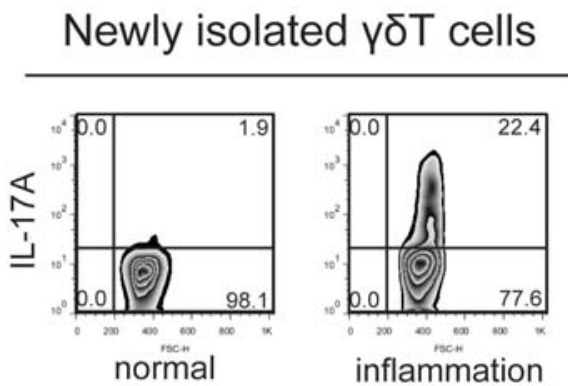

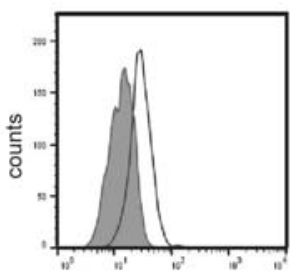

ICAM-1
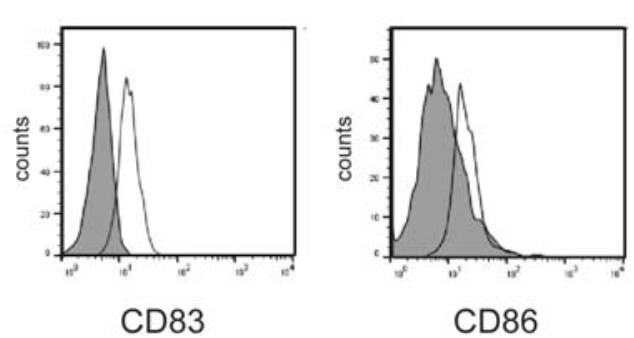

(d)

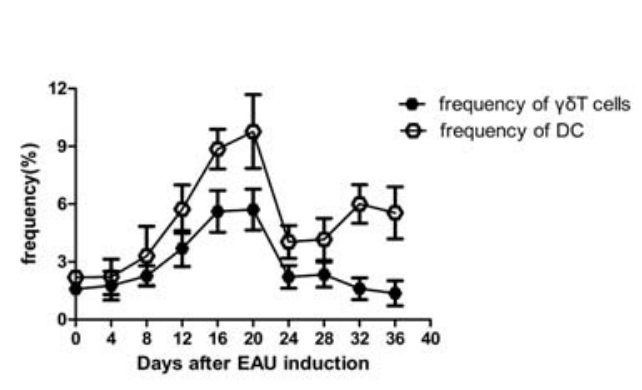

(e)

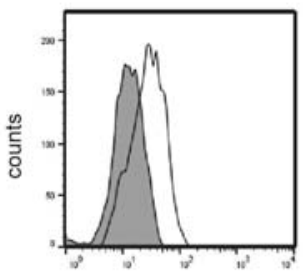

CD80

Fig. 2. The status of $\gamma \delta \mathrm{T}$ cells and DCs obtained from the spleens of immunized mice in the inflammatory phase, as analyzed using
flow cytometry. (a) The changes in the percentage of $\gamma \delta \mathrm{T}$ cells among splenic T-cells are shown in the left column. T-cells are
stained with PE-conjugated $\gamma \delta \mathrm{TCR}$ and APC-conjugated CD3e antibodies. T-cells that coexpressed $\gamma \delta \mathrm{TCR}$ and CD3e are $\gamma \delta \mathrm{T}$ exped $\gamma \delta$ TCR and CD3e are $\gamma \delta \mathrm{T}$ cells. The changes in the percentage of DCs among lymphocyte cells isolated from the spleens of mice are shown in the right column. (b) The expression of CD69 and LFA-1 in the $\gamma \delta$ T cells obtained from the spleens of normal mice (shadow line) compared with the expression in cells obtained from the spleens of mice with severe inflammatory symptoms (solid line). (c) The level of IL-17A in $\gamma \delta$ T cells obtained from the spleens of normal mice (left panel) and mice with severe inflammatory symptoms (right panel). (d) The expression of ICAM-1, CD80, CD86, and CD83 in DCs obtained from the spleens of normal mice (shadow line) compared with the expression in cells obtained from the spleens of immunized mice with severe inflammatory symptoms (solid line). (e) Changes in the percentages of $\gamma \delta \mathrm{T}$ cells and DCs obtained from the spleens of diseased mice on days $0,4,8,12,16,20,24,28,32$, and 36 after immunization.

molecule CD69 and LFA-1, which are markers of T-cell activation (Fig. 2(b)) and secreted greater amounts of IL-17A $(19.09 \% \pm 3.86 \%$ versus $1.88 \% \pm 0.28 \%$; Fig. 2(c), each figure shows a representative result of three experiments) than did the $\gamma \delta \mathrm{T}$ cells obtained from the normal mice. This indicated that the number of $\gamma \delta \mathrm{T}$ cells was increased in the inflammation phase, and that these 
cells were activated. In addition, the DCs from the immunized mice expressed higher levels of ICAM-1, CD80, CD83, and CD86 than did DCs from the normal mice (Fig. 2(d)). This indicated that the DCs had undergone maturation in the immunized mice. We also analyzed the changes in the percentages of $\gamma \delta \mathrm{T}$ cells and DCs obtained from the spleens of the immunized mice at different time points (days 0, 4, 8, 12, 16, 20, 24, 28, 32, and 36 after immunizations). The results showed that the percentages of both $\gamma \delta \mathrm{T}$ cells and DCs increased from day 8 , and peaked on days 16-day 20 . The above results show that the number of $\gamma \delta$ $\mathrm{T}$ cells and DCs increased during the inflammatory phase, and that the $\gamma \delta \mathrm{T}$ cells were activated and the DCs matured in the immunized mice. This suggested that both types of cells participated in the development of EAU. Moreover, we found that increase in the number of $\gamma \delta \mathrm{T}$ cells and DCs were associated with increased severity of EAU and both types of cells were activated in the inflammatory phase. This further indicated that activated $\gamma \delta \mathrm{T}$ cells and mature DCs participated in the progression of EAU. Then, the activated $\gamma \delta \mathrm{T}$ cells were transferred into the $\mathrm{IRBP}_{1-20}$ immunized mice. The symptom of the mice with $\gamma \delta \mathrm{T}$ cells injection was more aggravated than that of mice without $\gamma \delta \mathrm{T}$ cells injection (Supplementary Fig. 2(a)). However, the symptom of immunized $\gamma \delta$ TCR- $\delta^{-/}$mice was slighter than that in EAU model in the inflammatory phase (Supplementary Fig. 3).

\subsection{Co-culture of mature DCs and $\gamma \delta$ $T$ cells promoted $\gamma \delta T$ cell activation}

Pervious report showed that there is a crosstalk between DCs and $\gamma \delta \mathrm{T}$ cell, ${ }^{16,23-26}$ which could influent the activation of each other. Thus, we speculated the increasing of DCs in the process of EAU might correlate with the activation of $\gamma \delta \mathrm{T}$ cells. To analyze the relationship between DCs and $\gamma \delta \mathrm{T}$ cells, we isolated DCs from the spleens of immunized mice, induced their maturation, and pulsed them with $\mathrm{IRBP}_{1-20}$. Then, these mature DCs were transferred into the mice which were immunized by IRBP $_{1-20}$ after eight days. The inflammation symptoms in the eyes and the percentage of $\gamma \delta \mathrm{T}$ cells in the spleen were analyzed after transfer of DCs for four days. Increasingly severe symptom was found in mice with transfer of mature DCs. Supplementary Fig. 2(a) shows representative examples of histopathology without and with mature DCs injection. The percentage of $\gamma \delta \mathrm{T}$ cells from spleen increased and expressed high level CD69 and LFA-1 (Supplementary Figs. 2(b), 4(a) and 4(b)). However, the inflammation symptoms could not become aggravated and the percentage of $\gamma \delta \mathrm{T}$ cells was not changed, after immature DCs injection (supplementary Figs. 2(a) and 2(b)).

Furthermore, we isolated DCs from the spleens of immunized mice, induced their maturation, and pulsed them with $\operatorname{IRBP}_{1-20}$, and finally, co-cultured them with $\gamma \delta \mathrm{T}$ cells at a ratio of 1:1 in 24-well plates at $37^{\circ} \mathrm{C}$. After $24 \mathrm{~h}$, the cells were harvested,

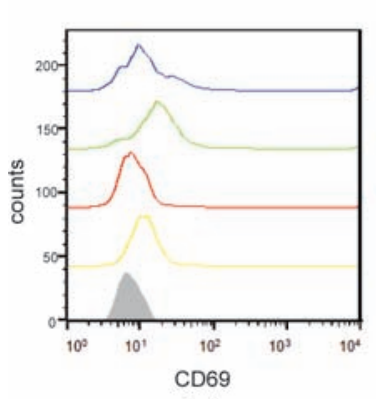

(a)

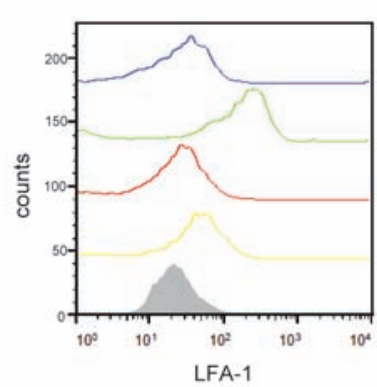

(b)

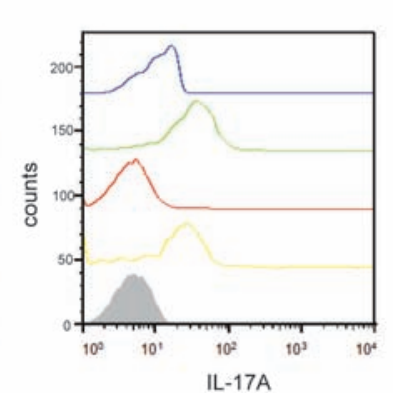

(c)

Fig. 3. (a) Activation of $\gamma \delta$ T cells co-cultured with DCs. (b) The $\gamma \delta$ T cells co-cultured with mature DCs expressed higher levels of activation markers (CD69, LFA-1; green line) than did $\gamma \delta$ T cells not cultured with DCs (shadow line), or that on the $\gamma \delta$ T cells cocultured with immature DCs (red line), or that on the $\gamma \delta$ T cells co-cultured with anti-ICAM-1 antibody pretreated DCs (blue line). (c) IL-17A expression is greater in $\gamma \delta \mathrm{T}$ cells co-cultured with mature DCs (green line) than in $\gamma \delta$ T cells cultured without DCs (shadow line), or that on the $\gamma \delta \mathrm{T}$ cells co-cultured with immature DCs (red line), or that on the $\gamma \delta$ T cells co-cultured with antiICAM-1 antibody pretreated DCs (blue line). 


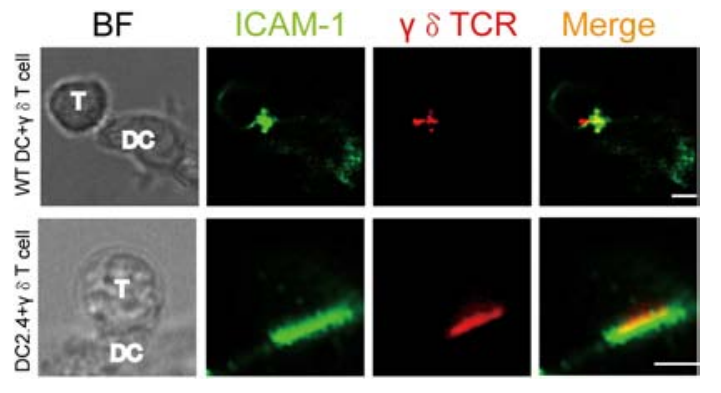

(a)

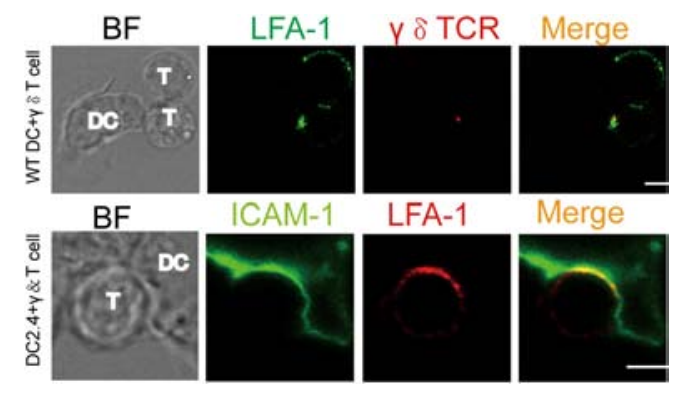

(b)

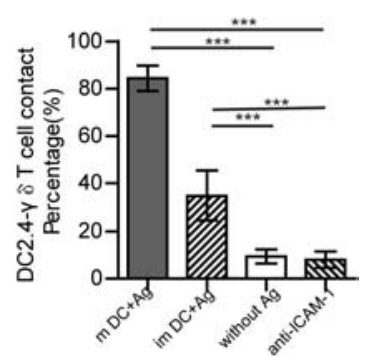

(c)

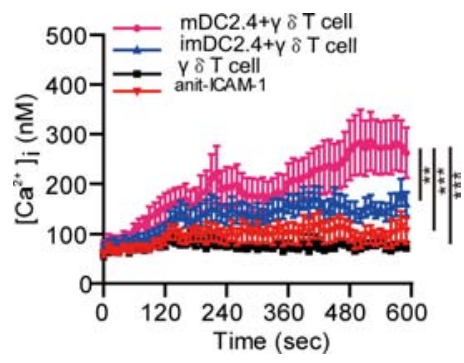

(d)

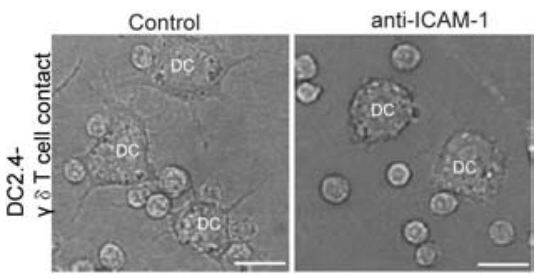

(e)

Fig. 4. The interaction between $\gamma \delta$ T cells and DCs. (a) The location of ICAM-1 and $\gamma \delta$ TCR at the interface between the DCs and $\gamma \delta \mathrm{T}$ cells. The upper line indicates the interaction between wild-type (WT) DCs and $\gamma \delta \mathrm{T}$ cells, and the $\gamma \delta \mathrm{T}$ cells were labeled with PE-conjugated $\gamma \delta$ TCR antibody (red), and DCs were labeled with APC-conjugated ICAM-1 antibody (green). The bottom line indicates the interaction between an ICAM-1-EGFP/DC2.4 cell and a $\gamma \delta$ T-cell. The $\gamma \delta \mathrm{T}$ cells were labeled with PE-conjugated $\gamma \delta$ TCR antibody (red). (b) The location of LFA-1 at the interface between the DCs and $\gamma \delta$ T cells. A $\gamma \delta$ T-cell interacts with a WT DC labeled with FITC-conjugated LFA-1 antibody (green) and PE-conjugated $\gamma \delta$ TCR antibody (red). A $\gamma \delta$ T-cell interacts with an ICAM-1-EGFP/DC2.4 cell (this cell line is a gift from professor Yiwei chu, ${ }^{40}$ Fudan university, Shanghai, China) labeled with PEconjugated LFA-1 antibody (red); the ICAM-1 on the DC 2.4 cell line is labeled with EGFP (green). The upper line indicates the interaction between a WT DC and a $\gamma \delta$ T-cell, and the bottom line indicates the interaction between an ICAM-1-EGFP/DC2.4 cell and a $\gamma \delta$ T-cell. (c) The amount of interaction between mature DC2.4 cell and $\gamma \delta \mathrm{T}$ cells and between immature DC2.4 cell and $\gamma \delta \mathrm{T}$ cells. The amount of interaction between DC2.4 cell and $\gamma \delta \mathrm{T}$ cells, without antigen. The amount of interaction between $\gamma \delta \mathrm{T}$ cells and DC2.4 cell with ani-ICAM-1 antibody pretreated. (d) Calcium signaling released in $\gamma \delta$ T cells interacting with mature DC2.4 cells, immature DC2.4 cells or pretreated DC2.4 cells. Data are expressed as mean \pm s.e.m.; ${ }^{* *} p<0.01,{ }^{* * *} p<0.001$ (two-tailed Student $t$-test). Scale bar $=2 \mu \mathrm{m}$. (e) The contact of DC- $\gamma \delta \mathrm{T}$ cell with or without anti-ICAM-1 antibody treatment. Scale bar $=10 \mu \mathrm{m}$.

and the status of the $\gamma \delta \mathrm{T}$ cells was determined using FACSVerse flow cytometry. The data showed that the $\gamma \delta \mathrm{T}$ cells co-cultured with mature DCs expressed higher levels of CD69 and LFA-1, and secreted higher levels of IL-17A than did $\gamma \delta \mathrm{T}$ cells not incubated with DCs (Figs. 3(a)-3(c)). In addition, immature DCs pulsed with $\mathrm{IRBP}_{1-20}$ could not induce the increase in LFA-1 expression and IL17A secretion by $\gamma \delta \mathrm{T}$ cells (Figs. 3(a)-3(c)). Moreover, $\gamma \delta \mathrm{T}$ cells co-cultured with mature DCs were induced a slight increase in LFA-1 expression and IL-17A secretion than activated $\gamma \delta \mathrm{T}$ cells did, which was stimulated by cytokines in vitro, although activated $\gamma \delta \mathrm{T}$ cells expressed higher levels of CD69 and LFA-1, and secreted higher levels of IL-17A than did $\gamma \delta \mathrm{T}$ cells not incubated with DCs (Figs. 3(a)-3(c)). These data indicated that DCs might promote the activation of $\gamma \delta \mathrm{T}$ cells in vitro. The mature DCs possessed a much stronger ability to activate $\gamma \delta \mathrm{T}$ cells than did the immature DCs.

Furthermore, DCs-pretreated $\gamma \delta \mathrm{T}$ cells or $\gamma \delta$ $\mathrm{T}$ cells-pretreated DCs were transferred into the $\mathrm{IRBP}_{1-20}$ immunized mice. The symptoms of those mice were more aggravated and the percentage of DCs or $\gamma \delta \mathrm{T}$ cells was higher than that of mice without cell injection (Supplementary Figs. 2(a)2(c), and Supplementary Fig. 4). In addition, a DC was found to contact with two or more $\gamma \delta$ T cells in the spleen of immunized mice (Supplementary Fig. 5). Thus, the interaction of DCs and $\gamma \delta \mathrm{T}$ cells might aggravate the symptoms of EAU. 


\subsection{ICAM-1 and $\gamma \delta$ TCR accumulated at the interface of DCs and $\gamma \delta$ $T$ cells}

To further analyze how DCs promoted $\gamma \delta$ T-cell activation, we isolated DCs and $\gamma \delta \mathrm{T}$ cells from the spleens of EAU mice at 16 days after immunization. We then pulsed the DCs with $\mathrm{IRBP}_{1-20}$ and co-cultured them with $\gamma \delta \mathrm{T}$ cells at a 1:1 ratio in 24 -well plates at $37^{\circ} \mathrm{C}$. A confocal system was used to measure the interaction between the DCs and $\gamma \delta$ T cells. For this analysis, we labeled the $\gamma \delta$ TCR of $\mathrm{CD} 4{ }^{+} \mathrm{T}$ cells with PE-conjugated $\gamma \delta$ TCR antibody in order to mark the locations of TCR clusters in $\mathrm{CD}^{+}{ }^{+} \mathrm{T}$ cells. Similarly, we labeled DCs with APCconjugated ICAM-1 antibody to indicate ICAM-1 clusters. Our data showed that ICAM- 1 and $\gamma \delta$ TCR accumulated at the interface between the mature DCs and $\gamma \delta \mathrm{T}$ cells obtained from the immunized mice (Fig. 4(a), upper line). Furthermore, we found that LFA- 1 and $\gamma \delta$ TCR were co-located at the interface of mature DCs and $\gamma \delta \mathrm{T}$ cells (Fig. 4(b), upper line). The same results were found in the case of the IRBP ${ }_{1-20}$-pulsed DC2.4-ICAM-1GFP cell line and $\gamma \delta$ T cells (Figs. 4(a) and 4(b) bottom line). Both mature and immature DCs could interact with $\gamma \delta \mathrm{T}$ cells, but the interaction was more frequently found at mature $\mathrm{DCs}-\gamma \delta \mathrm{T}$ cells than immature DCs $-\gamma \delta$ T cells $(84.3 \pm 5.36 \%$ versus $35 \pm 10.48 \%$ ) (Fig. 4(c)). Moreover, IRPB $_{1-20}$ pulsed immature DCs contacted $\gamma \delta \mathrm{T}$ could accumulate ICAM- 1 and $\gamma \delta$ TCR at the interface of DC and $\gamma \delta \mathrm{T}$ cell (date not shown). Thus, the DCs from the diseased mice could preferentially interact with $\gamma \delta$ T cells. The accumulation of LFA- 1 and ICAM- 1 at the interface between the DCs and $\gamma \delta \mathrm{T}$ cells might be beneficial for $\mathrm{DC}-\gamma \delta \mathrm{T}$-cell interaction.

Calcium release is a marker of T-cell activation. To determine whether the $\mathrm{DC}-\gamma \delta \mathrm{T}$-cell interaction promoted the activation of $\gamma \delta \mathrm{T}$ cells, we measured the calcium release from $\gamma \delta \mathrm{T}$ cells interacting with DCs. By imaging analysis, we found that $\gamma \delta \mathrm{T}$ cells in contact with mature DCs released higher levels of calcium than did cells that were in contact with immature DCs (Fig. 4(d)). This suggested that the $\mathrm{DC}-\gamma \delta \mathrm{T}$ cell interaction might be a factor to contribute the activation of $\gamma \delta$ T-cell. Furthermore, mature DCs might play a major role in the interaction of DCs with $\gamma \delta \mathrm{T}$ cells to promote the activation of the latter.

\subsection{Blocking the interaction of $D C-\gamma \delta$ $T$-cell, the activation of $\gamma \delta$ T-cell was decreased}

To confirm that $\gamma \delta$ T-cell activation was promoted by the $\mathrm{DC}-\gamma \delta$ T-cell interaction, we blocked this interaction by using anti-ICAM-1 antibody. We found the expression of LFA-1, CD69, and IL-17A in $\gamma \delta \mathrm{T}$ cells in contact with the treated DCs which did not significantly differ from the expression in $\gamma \delta$ $\mathrm{T}$ cells not in contact with DCs (Figs. 3(a)-3(c)). The contact between the DCs and $\gamma \delta \mathrm{T}$ cells was decreased by the anti-ICAM-1 antibody (Figs. 4(c) and $4(\mathrm{e}))$. The calcium response was decreased in $\gamma \delta$ $\mathrm{T}$ cells contacted treated DCs, as compared to $\gamma \delta \mathrm{T}$ cells cultured with mature DCs (Fig. 4(d)).

In addition, we pretreated the mature DCs with anti-ICAM-1 antibody to block the binding of DCs and $\gamma \delta \mathrm{T}$ cells and transferred those DCs to immunized mice. We found the symptom of the mice with transfer of ICAM-1 antibody pretreated DCs was slighter and the percentage of activated $\gamma \delta \mathrm{T}$ cells decreased, comparing mice with transfer of mature DCs (Supplementary Figs. 2(a) and 2(b), Supplementary Fig. 4). It indicated blocking the contact sites of DCs and $\gamma \delta$ T cells, the activated $\gamma \delta$ $\mathrm{T}$ cells decreased.

\section{Discussion}

It was previously believed that $\gamma \delta \mathrm{T}$ cells bridge the gap between innate and adaptive immunity ${ }^{27-29}$ and regulate the intensity of adaptive immune responses. ${ }^{30,31}$ These cells are increasingly being recognized as an important source of IL-17A in several autoimmune diseases. ${ }^{32-34}$ The cells might reside in the target organ, where they incite a rapid immune response upon activation even before naive $\mathrm{CD}_{4}^{+} \mathrm{T}$ cells detect their cognate antigens in the secondary lymphoid organs and enhance the development of IL-17A-producing $\alpha \beta \mathrm{T}$ cells, thereby damaging the target organ and aggravating the autoimmune disease. ${ }^{35,36} \mathrm{In}$ a previous report, $\gamma \delta \mathrm{T}$ cells were found to represent a critical element of the autoreactive responses involved in $\mathrm{IRBP}_{1-20^{-}}$ induced EAU in B 6 mice. ${ }^{11}$ The activation of $\gamma \delta \mathrm{T}$ cells is believed to promote the development of EAU. ${ }^{10,11,37}$ Moreover, the proinflammatory effect of $\gamma \delta \mathrm{T}$ cells was found to be augmented by their activation. $^{10,11}$ However, the mechanism of $\gamma \delta$ 
T-cells activation has not been reported. In the present study, we found that the DCs, especially mature DCs activated $\gamma \delta \mathrm{T}$ cells, and the interaction of DCs and $\gamma \delta \mathrm{T}$ cells might be a factor to promote the activation of $\gamma \delta \mathrm{T}$ cells in EAU.

Firstly, we measured the dynamic change of $\gamma \delta \mathrm{T}$ cells following with EAU progression, and then transferred the activated $\gamma \delta \mathrm{T}$ cells into EAU model, the symptoms of EAU were aggravated. However, the symptoms of immunized $\gamma \delta$ TCR$\delta^{-/-}$mice were relieved obviously. Thus, activated $\gamma \delta \mathrm{T}$ cells play a pathogenic role in EAU.

Furthermore, mature DCs were transferred into EAU model, the symptom of EAU was aggravated and the percentage of activated $\gamma \delta \mathrm{T}$ cells increased. However, the transfer of immature DCs did not aggravate the symptoms of EAU. In vitro experiment, we found that mature DCs promoted higher level of CD69 and LFA-1 expression and IL-17A secretion in the $\gamma \delta \mathrm{T}$ cells co-cultured with DCs than that in activated $\gamma \delta \mathrm{T}$ cells, which activated by cytokines or that in resting $\gamma \delta \mathrm{T}$ cells. Thus, mature DCs might promote the activation of $\gamma \delta \mathrm{T}$ cells, and the ability of activating $\gamma \delta \mathrm{T}$ cells of this process was much stronger than that of antigen and cytokine stimulation in vitro.

To further analyze whether mature DCs promote the activation of $\gamma \delta \mathrm{T}$ cells through interacting with $\gamma \delta \mathrm{T}$ cells, we measure the interaction of DCs and $\gamma \delta$ $\mathrm{T}$ cells by confocal and flow cytometry. By imaging study, the contact formed between DCs and $\gamma \delta$ $\mathrm{T}$ cell was found in vitro and ex vivo. Blocking the contact of DCs $-\gamma \delta$ T cells by anti-ICAM- 1 antibody, the activation of $\gamma \delta$ T cells was decreased. In addition, the transfer of DCs-pretreated $\gamma \delta$ $\mathrm{T}$ cells or $\gamma \delta \mathrm{T}$ cells-pretreated DCs aggravated the symptom of EAU. Thus, the interaction of DCs and $\gamma \delta \mathrm{T}$ cells might be a factor to affect the activation of $\gamma \delta \mathrm{T}$ cells, leading to the aggravated symptom of EAU. Whether the interaction between DCs and $\gamma \delta$ $\mathrm{T}$ cell could affect the mature of DC to influence the development of EAU, this problem would be another interesting problem for us to further study in the future.

Crosstalk between $\gamma \delta \mathrm{T}$ cells and DCs via immunological synapse formation has been reported. ${ }^{38}$ An immunological synapse is a platform formed between a T-cell and an APC. Multi-molecular activation clusters, involving TCR-MHC, ICAM-1LFA-1, CD28-CD80, etc., accumulate at the T-cell-APC interface. ${ }^{39}$ The immunological synapse can control T-cell activation and regulate immune responses. ${ }^{39,40}$ In our study, ICAM-1, LFA-1, and $\gamma \delta$ TCR were found to be localized at the interface between DCs and $\gamma \delta \mathrm{T}$ cells obtained from EAU mice. This indicated that immunological synapses were formed between the DCs and $\gamma \delta$ T cells. Such synapses might promote the crosstalk between DCs and $\gamma \delta \mathrm{T}$ cells. The activation and movement of LFA-1 follows the movement of ICAM-1, and the binding of ICAM-1 and LFA-1 promotes contact between the two cells. ${ }^{41}$ Thus, ICAM-1-LFA-1 binding might be helpful for contact between DCs and $\gamma \delta \mathrm{T}$ cells. The mechanism underlying the activation of $\gamma \delta \mathrm{T}$ cells by DCs in EAU required further investigation.

We studied the mechanism of $\gamma \delta$ T-cell activation by flow cytometry and confocal microscopy. On flow cytometry, we found that the co-culture of $\gamma \delta$ $\mathrm{T}$ cells with mature DCs promoted the expression of CD69 and LFA-1 on $\gamma \delta \mathrm{T}$ cells. Using imaging studies, we proved the contact between DCs and $\gamma \delta$ $T$ cells and detected the accumulation of LFA-1 and ICAM- 1 at the interface between the DCs and $\gamma \delta \mathrm{T}$ cells. Furthermore, by cell transfer and the cell-cell contact imaging, the interaction of DCs and $\gamma \delta \mathrm{T}$ cells might be a factor to promote the development of EAU through activating $\gamma \delta \mathrm{T}$ cells. More detailed imaging studies will provide further information about immune responses and the molecular mechanism of $\mathrm{DC}-\gamma \delta \mathrm{T}$ cell contact.

\section{Acknowledgments}

We are grateful to Professor yiwei Chu (Fudan university, shanghai, China) for giving DC2.4ICAM-1-GFP cell line as a gift. This work was supported by the National Natural Science Foundation of China (81373826, 81403438 and 81500710).

\section{References}

1. A. Rothova, V. S. Suttorp, T. Frits, A. Kijlstra, "Causes and frequency of blindness in patients with intraocular inflammatory disease," Br. J. Ophthalmol. 80, 332-336 (1996).

2. K. W. Wucherpfennig, J. Newcombe, H. Li, C. Keddy, M. L. Cuzner, D. A. Hafler, " $\gamma \delta$ T-cell receptor repertoire in acute multiple sclerosis lesions," Proc. Natl. Acad. Sci. 89(10), 4588-4592 (1992). 
3. S. L. Peng, M. P. Madaio, A. C. Hayday, J. Craft, "Propagation and regulation of systemic autoimmunity by $\gamma \delta$ T cells," J. Immunol. 157(12), 56895698 (1996).

4. T. W. Spahn, S. Issazadah, A. J. Salvin, H. L. Weiner, "Decreased severity of myelin oligodendrocyte glycoprotein peptide 33-35-induced experimental autoimmune encephalomyelitis in mice with a disrupted TCR $\delta$ chain gene," Eur. J. Immunol. 29(12), 4060-4071 (1999).

5. A. J. Rajan, V. C. Asensio, I. L. Campbell, C. F. Brosnan, "Experimental autoimmune encephalomyelitis on the SJL mouse: Effect of $\gamma \delta \mathrm{T}$ cell depletion on chemokine and chemokine receptor expression in the central nervous system," $J$. Immunol. 164(4), 2120-2130 (2000).

6. A. Odyniec, M. Szczepanik, M. P. Mycko, M. Stasiolek, C. S. Raine, K. W. Selmaj, " $\gamma \delta$ T cells enhance the expression of experimental autoimmune encephalomyelitis by promoting antigen presentation and IL-12 production," J. Immunol. 173(1), 682-694 (2004).

7. K. Uezu, K. Kawakami, K. Miyagi, Y. Kinjo, T. Kinjo, H. Ishikawa, A. Saito, "Accumulation of $\gamma \delta \mathrm{T}$ cells in the lungs and their regulatory roles in Th1 response and host defense against pulmonary infection with Cryptococcus neoformans," J. Immunol. 172(12), 7629-7634 (2004).

8. C. D. D'Souza, A. M. Cooper, A. A. Frank, R. J. Mazzaccaro, B. R. Bloom, I. M. Orme, "An antiinflammatory role for $\gamma \delta \mathrm{T}$ lymphocytes in acquired immunity to Mycobacterium tuberculosis," J. Immunol. 158(3), 1217-1221 (1997).

9. W. Born, C. Cady, J. Jones-Carson, A. Mukasa, M. Lahn, R. O'Brien, "Immunoregulatory functions of $\gamma \delta$ T cells," Adv. Immunol. 71, 77-144 (1999).

10. H. Nian, H. Shao, R. L. O'Brien, W. K. Born, H. J. Kaplan, D. Sun, "Activated gammadelta $\mathrm{T}$ cells promote the activation of uveitogenic $\mathrm{T}$ cells and exacerbate EAU development," Invest. Ophthalmol. Vis. Sci. 52(8), 5920-5927 (2011).

11. Y. Cui, H. Shao, C. Lan, H. Nian, R. L. O'Brien, W. K. Born, H. J. Kaplan, D. Sun, "Major role of $\gamma \delta \mathrm{T}$ cells in the generation of IL-17 uveitogenic T cells," J. Immunol. 183(1), 560-567 (2009).

12. R. M. Steinman, J. Banchereau, "Taking dendritic cells into medicine," Nature 449(7161), 419-426 (2007).

13. R. M. Steinman, "Lasker Basic Medical Research Award Dendritic cells: Versatile controllers of the immune system," Nat. Med. 13(10), 1155-1159 (2007).

14. G. De Libero, L. Mori, "Recognition of lipid antigens by T cells," Nat. Rev. Immunol. 5(6), 485-496 (2005).
15. S. M. Behar, S. A. Porcelli, "CD1-restricted T cells in host defense to infectious diseases," Curr. Top. Microbiol. Immunol. 314, 215-250 (2007).

16. F. Miyagawa, Y. Tanaka, S. Yamashita, "Essential requirement of antigen presentation by monocyte lineage cells for the activation of primary human gamma delta $\mathrm{T}$ cells by aminobisphophonate antigen," J. Immunol. 166(9), 5508-5514 (2001).

17. M. C. Devilder, S. Maillet, I. Bouyge-Moreau, E. Donnadieu, M. Bonneville, E. Scotet. "Potentiation of antigen-stimulated $\mathrm{V}$ galnma $9 \mathrm{~V}$ delta $2 \mathrm{~T}$ cell cytokine production by immature dendrifle cells (DC)and reciprocal effect on DC maturation," J. Immunol. 176(3), 1386-1393 (2006).

18. K. Inaba, S. Turley, T. Iyoda, F. Yamaide, S. Shimoyama, C. Reis e Sousa, R. N. Germain, I. Mellman, R. M. Steinman, "The formation of immunogenic major histocompatibility complex class II-peptide ligands in lysosomal compartments of dendritic cells is regulated by inflammatory stimuli," J. Exp. Med. 191(6), 927-936 (2000).

19. S. Fujii, K. Liu, C. Smith, A. J. Bonito, R. M. Steinman, "The linkage of innate to adaptive immunity via maturing dendritic cells in vivo requires CD40 ligation in addition to antigen presentation and CD80/86 costimulation," J. Exp. Med. 199(12), 1607-1618 (2004).

20. N. D. Heuss, U. Lehmann, C. C. Norbury, S. W. McPherson, D. S. Gregerson, "Local Activation of Dendritic Cells Alters the Pathogenesis of Autoimmune Disease in the Retina," J. Immunol. 188(3), 1191-1200 (2012).

21. P. Chen, A. K. Denniston, S. Hirani, S. Hannes, R. B. Nussenblatt, "Role of dendritic cell subsets in immunity and their contribution to noninfectious uveitis," Surv. Ophthalmol. 60(3), 242-249 (2015).

22. S. R. Thurau, C. C. Chan, R. B. Nussenblatt, R. R. Caspi, "Oral tolerance in a murine model of relapsing experimental autoimmune uveoretinitis (EAU): Induction of protective tolerance in primed animals," Clin. Exp. Immunol. 109(2), 370-376 (1997).

23. D. Liang, A. Zuo, H. Shao, W. K. Born, R. L. O'Brien, H. J. Kaplan, D. Sun, "Role of CD25+ dendritic cells in the generation of Th17 autoreactive $\mathrm{T}$ cells in autoimmune experimental uveitis," J. Immunol. 188(11), 5785-5791 (2012).

24. D. S. Leslie, M. S. Vincent, F. M. Spada, H. Das, M. Sugita, C. T. Morita, M. B. Brenner, "CD1-mediated gamma/delta $\mathrm{T}$ cell maturation of dendritic cells," J. Exp. Med. 196(12), 1575-1584 (2002).

25. C. Collins, J. Wolfe, K. Roessner, C. Shi, L. HSigal, R. C. Budd, "Lyme arthritis synovial gammadelta T cells instruct dendritic cells via fas ligand," $J$. Immunol. 175(9), 5656-5665 (2005). 
26. C. Munz, R. M. Steinman, S. Fujii, "Dendritic cell maturation by innate lymphocytes: Coordinated stimulation of innate and adaptive immunity," $J$. Exp. Med. 202(2), 203-207 (2005).

27. Y. X. Fu, C. E. Roark, K. Kelly, D. Drevets, P. Campbell, R. O'Brien, W. Born, "Immune protection and control of inflammatory tissue necrosis by $\gamma \delta$ T cells," J. Immunol. 153(7), 3101-3115 (1994).

28. T. A. Moore, B. B. Moore, M. W. Newstead, T. J. Standiford, " $\gamma \delta \mathrm{T}$ cells are critical for survival and early proinflammatory cytokine gene expression during murine Klebsiella pneumonia," J. Immunol. 165(5), 2643-2650 (2000).

29. C. Nakasone, N. Yamamoto, M. Nakamatsu, T. Kinjo, K. Miyagi, K. Uezu, K. Nakamura, F. Higa, H. Ishikawa, R. L. O’brien, K. Ikuta, M. Kaku, J. Fujita, K. Kawakami, "Accumulation of $\gamma \delta \mathrm{T}$ cells in the lungs and their roles in neutrophil-mediated host defense against pneumococcal infection," Microbes. Infect. 9(3), 251-258 (2007).

30. P. Mombaerts, J. Arnoldi, F. Russ, S. Tonegawa, S. H. Kaufmann, "Different roles of $\alpha \beta$ and $\gamma \delta \mathrm{T}$ cells in immunity against an intracellular bacterial pathogen," Nature 365(6441), 53-56 (1993).

31. R. K. Braun, C. Ferrick, P. Neubauer, M. Sjoding, A. Sterner-Kock, M. Kock, L. Putney, D. A. Ferrick, D. M. Hyde, R. B. Love, "IL-17 producing $\gamma \delta$ T cells are required for a controlled inflammatory response after bleomycin-induced lung injury," Inflammation 31(3), 167-179 (2008).

32. C. L. Roark, J. D. French, M. A. Taylor, A. M. Bendele, W. K. Born, R. L. O'Brien, "Exacerbation of collagen-induced arthritis by oligoclonal, IL-17producing gamma delta T cells," J. Immunol. 179 (8), 5576-5583 (2007).

33. C. E. Sutton, S. J. Lalor, C. M. Sweeney, C. F. Brereton, E. C. Lavelle, K. H. Mills, "Interleukin-1 and IL-23 induce innate IL-17 production from gammadelta T cells, amplifying Th17 responses and autoimmunity," Immunity 31(2), 331-341 (2009).

34. F. Petermann, V. Rothhammer, M. C. Claussen, J. D. Haas, L. R. Blanco, S. Heink, I. Prinz, B. Hemmer, V. K. Kuchroo, M. Oukka, T. Korn, " $\gamma \delta$ T cells enhance autoimmunity by restraining regulatory $\mathrm{T}$ cell responses via an interleukin-23-dependent mechanism," Immunity 33(3), 351-363 (2010).

35. D. Kabelitz, D. Wesch, W. He, "Perspectives of gammadelta T cells in tumor immunology," Cancer Res. 67(1), 5-8 (2007).

36. M. Girardi, "Immunosurveillance and immunoregulation by gammadelta T cells," J. Invest. Dermatol. 126(1), 25-31 (2006).

37. H. Nian, H. Shao, G. Zhang, W. K. Born, R. L. O'Brien, H. J. Kaplan, D. Sun, "Regulatory effect of T cells on IL-17 uveitogenic T cells," Invest. Ophthalmol. Vis. Sci. 51(9), 4661-4667 (2010).

38. J. M. Wands, C. L. Roark, M. K. Aydintug, N. Jin, Y. S. Hahn, L. Cook, X. Yin, J. Dal Porto, M. Lahn, D. M. Hyde, E. W. Gelfand, R. J. Mason, R. L. O'Brien, W. K. Born. "Distribition and leukocyte contacts of gammadelta T cells in the lung," J. Leukoc. Biol. 78(5), 1086-1096 (2005).

39. A. Grakoui, S.K. Bromley, C. Sumen, M.M, Davis, A. S. Shaw, P. M. Allen, M. L. Dustin, "The immunological synapse: A molecular machine controlling T cell activation," Science 285, 221-227 (1999).

40. W. Lin, Z. Fan, Y. Suo, Y. Deng, M. Zhang, J. Wang, X. Wei, Y. Chu, "The bullseye synapse formed between CD4+ T-cell and staphylococcal enterotoxin B-pulsed dendritic cell is a suppressive synapse in T-cell response," Immunol. Cell Biol. 93, 99-110 (2015).

41. W. A. Comrie, S. Li, S. Boyle, J. K. Burkhardt, "The dendritic cell cytoskeleton promotes $\mathrm{T}$ cell adhesion and activation by constraining ICAM-1 mobility," J. Cell Biol. 208(4), 457-473 (2015). 